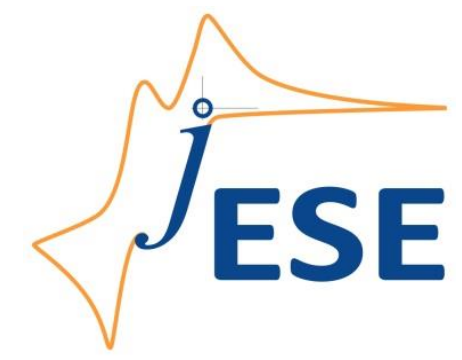

Open Access : : ISSN 1847-9286

www.jESE-online.org

Original scientific paper

\title{
Spectroscopic, voltammetry and molecular docking study of binding interaction of antipsychotic drug with bovine serum albumin
}

\author{
Mallappa Mahanthappa, Babu Giriya Gowda ${ }^{\bowtie}$, Jayant I. Gowda*, Raghavendran \\ Rengaswamy
}

Department of Chemistry, Maharani's Science College for Women, Bangalore-560 001, India

*P.G. Department of Studies in Chemistry, Karnatak University, Dharwad- 580 003, India

${ }^{\square}$ Corresponding Author: babgowda@gmail.com, Phone: +91-9900475412

Received: July 22, 2015; Accepted: February 4, 2016

\begin{abstract}
The interaction between perazine dimaleate (PDM) and bovine serum albumin (BSA) was investigated by voltammetry, fluorescence spectroscopy, UV-vis spectroscopy, molecular docking and viscometric methods. The study was carried out in acetate buffer solution of pH 7.2, which was prepared by using $0.1 \mathrm{M}$ sodium acetate and adjusting $\mathrm{pH}$ using $0.1 \mathrm{M}$ hydrochloric acid. The voltammetric study of PDM shows a pair of well redox peaks at 0.538 and $0.471 \mathrm{~V}$ (versus SCE) on a GCE in acetate buffer of $p H 7.2$ at $50 \mathrm{mV} \mathrm{s}^{-1}$. After the addition of BSA into the PDM solution, the redox peak currents decreased gradually, and peak potentials shifted towards negative direction. The results of voltammetry, fluorescence quenching and UV-vis absorption spectra experiments indicated the formation BSA-PDM complex. The binding parameters like binding constant and binding free energy were determined from voltammetric data. The binding constant and binding energy was also determined from UV-vis and fluorescence spectroscopy with a value quite close to that obtained from CV.
\end{abstract}

\section{Keywords}

Bovine serum albumin; Perazine dimaleate; Cyclic Voltammetry; Spectroscopy; Molecular docking; Binding Constant; Binding energy

\section{Introduction}

The drug-protein interaction is a fundamental issue in life process that helps us to understand the absorption, transport, metabolism and the target molecules of the drugs at the cellular level 
[1-3]. Serum albumins are the major soluble protein constituents of the circulatory system possessing many physiological functions of which the most important are serving as a depot and a transport protein for many endogenous and exogenous compounds such as drugs [4]. The interaction of drugs with serum albumin is great aspect and various types of application in research field in chemistry, life science and clinical medicine [5-8]. Bovine serum albumin (BSA) has been widely investigated due to its structural homology with human serum albumin [9]. Intercalation, groove binding, and electrostatic interactions are the three major binding modes of small molecules to BSA [10].

Perazine dimaleate (PDM) is biologically active drug, chemically known as 10-[3-(4 methylpiperazine-1-yl)propyl]-10-phenothiazine-2maleicacid; perazine maleate; Pernazinum (Fig. 1). PDM is important class of tricyclic compounds belongs to the phenothiazine family drugs. Generally, phenothiazine drugs are widely used as antiemetic, sedative and analgesic because they are able to reduce the side effects of chemotherapy and they they also have a role in inhibiting tumor cell proliferation. These drugs can also reduce anxiety, insomnia and other symptoms related to the psychological state of cancer patients [11].

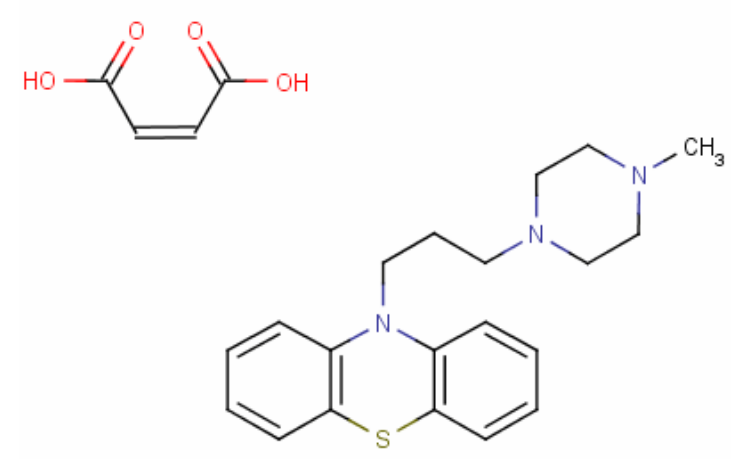

Fig. 1. Chemical structure of perazine dimaleate (PDM)

There has not any report about the study of PDM-BSA interaction based on the voltammetry behaviours at GCE and more than ever on the change of spectroscopic characteristics. Accordingly, in this work, investigations of the voltammetric behaviour of PDM upon addition of BSA were carried out. Moreover, the changes in the UV-Vis absorption and fluorescence emission when PDM binding to BSA were used to study the mode of such interaction. The results of voltammetry and spectroscopic techniques confirmed each other. The interaction study of PDM with BSA gives the information about the quenching mechanisms, binding constant, binding mode and number of binding sites. The advantage of this method is simple, rapid, accurate and inexpensive.

\section{Materials and methods}

PDM sample was donated by Global Calcium Ltd., Hosur. Stock solution, $1.0 \mathrm{mmol} \mathrm{L}^{-1}$ of PDM was prepared by dissolving an appropriate amount of the drug in doubly distilled water. BSA (Sigma-Aldrich) was used without further purification and its stock solution was prepared by dissolving an appropriate amount of BSA in doubly distilled water and stored at $4{ }^{\circ} \mathrm{C}$. The concentration of BSA in stock solution was determined by UV absorption at $260 \mathrm{~nm}$ using a molar absorption coefficient $\varepsilon_{260}=66,000 \mathrm{~L} \mathrm{~mol}^{-1} \mathrm{~cm}^{-1}$. Purity of the BSA was checked by monitoring the ratio of the absorbance at $260 \mathrm{~nm}$ to that at $280 \mathrm{~nm}$ [12]. The supporting electrolyte used for $0.1 \mathrm{M}$ acetate buffer solution of $\mathrm{pH} 7.2$, which was prepared by using $0.1 \mathrm{M}$ sodium acetate and $\mathrm{pH}$ was adjusted by using $0.1 \mathrm{M}$ acetic acid and all the experiments were conducted in this buffer 
at $25^{\circ} \mathrm{C}$. All other reagents used were of analytical grade and doubly distilled water was used throughout the experiment.

\section{Instrumentation}

Voltammetric measurements were performed using a $\mathrm{CH}$ electrochemical analyzer. A conventional three-electrode cell was employed throughout the experiments, with glassy carbon electrode ( $3.0 \mathrm{~mm}$ diameter) as a working electrode, a saturated calomel electrode (SCE) as a reference electrode, and a platinum electrode as a counter electrode.

Absorption spectra were measured on Ellico UV-visible spectrophotometer. The electronic spectrum of a known concentration of the drug was obtained without BSA. The spectroscopic response of the same amount of the drug was then monitored by the addition of small aliquots of BSA solution. All of the samples were mixed properly and allowed to equilibrate for 5 min prior to every spectroscopic measurement.

The viscosity measurements were carried out using Ostwald viscometer. The $\mathrm{pH}$ measurements were made with Scott Gerate pH meter CG 804. An electronic thermostat water-bath was used for controlling the temperature.

The steady-state fluorescence spectra were recorded using an F-2700 spectrofluorometer (Japan). The fluorescence emission spectrum of PDM (excitation at $250 \mathrm{~nm}$ ) was used to obtain the intensities of the peaks. Good resolution of the bands was obtained at the slit width (ex. $10.0 \mathrm{~nm}$; em.10.0 nm). The scan range used was from 300 to $600 \mathrm{~nm}$.

The molecular docking studies of BSA with compounds were achieved by using Auto dock Vina, developed at the Scripps research institute (http://vina.scripps.edu) [13]. The input files for AutoDock Vina were prepared with AutoDock Tools (ADT), which is a Graphical User Interface for AutoDock and AutoDock Vina. The crystal structure of bovine serum albumin (3V03) was retrieved from Protein data bank and the ligand binding site location was analyzed by Q-Site Finder [14]. The co-crystallized ligand was removed. Using ADT the water molecules were removed from the protein and polar hydrogen were added followed by adding Kollman charges. The Grid box has been set according to the binding site on protein and saved as pdbqt format, which was required by AutoDock Vina. The 2 dimensional structure of ligand were drawn by Chem sketch and converted to PDB format, required by AutoDock Tools by Open Babel [15]. The rotatable bonds were selected within the ligand using Choose Torsions option in ADT and saved in pdbqt format The Lamarckian Genetic Algorithm (LGA), which is a novel and robust automated docking method available in AutoDock [16], was employed.

\section{Results and discussion}

\section{Voltammetric studies of interaction of PDM with BSA}

The Voltammetric behaviour of $1.5 \times 10^{-4} \mathrm{M}$ PDM in the absence and presence of BSA on bare GCE at $50 \mathrm{mV} \mathrm{s}^{-1}$ is shown in Fig. 2. The voltammogram without BSA featured a couple of well defined and stable redox peaks in the potential range of $0.0-1.0 \mathrm{~V}$. The anodic and cathodic peaks were appeared at 0.538 and $0.471 \mathrm{~V}$ vs. SCE with a formal potential $\left(E^{0}\right)$ of $0.5045 \mathrm{~V}$. The formal potential $\left(E^{\circ}\right)$ in all voltammetric studies, predictable as the midpoint of reduction and oxidation potentials. By the addition of $5-30 \mu \mathrm{mol} \mathrm{L}^{-1}$ of BSA into $1.5 \times 10^{-4} \mathrm{M}$ drug (Fig. 2) the anodic peak potential was shifted towards negative direction and also peak current was decreased. The substantial decrease in peak current indicates the formation of PDM-BSA complex. 


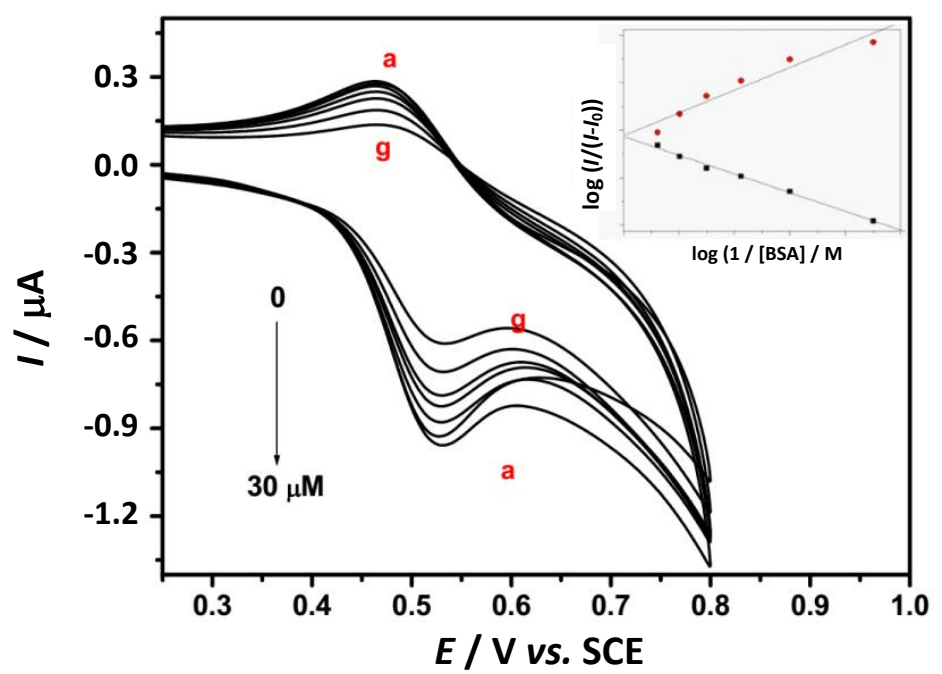

Fig. 2. Cyclic voltammogram of $1.5 \times 10^{-4} \mathrm{M} P D M$ in the absence of BSA and in presence of $C_{B S A}=0,5,10,15,20,25,30 \mu \mathrm{mol} \mathrm{L}^{-1}$ (a to g) BSA in acetate buffer of $\mathrm{pH}-7.2$ at $50 \mathrm{mV} \mathrm{s}^{-1}$ and inset of the plot of $\log (1 /[B S A])$ vs. $\log \left(\mathrm{I} /\left(\mathrm{I}_{0}-1\right)\right)$.

The mode of PDM-BSA interaction can be well-known from the variation in formal potential. In general the positive shift (anodic shift) in formal potential is caused by the intercalation of the drug with BSA [17], while negative shift is observed for the electrostatic interaction of the drug with BSA [18]. Therefore, this is evident that negative peak potential shift (cathodic shift) in the CV behaviour of PDM by the addition of BSA is attributable to the electrostatic interaction of PDM with BSA. The cathodic peak potential shift further indicates that PDM is easier to oxidize in presence of BSA because its oxidized form is more strongly bound to BSA than its reduced form. In this system, both forms of the drug interact with BSA.

Based upon the decrease in peak current of PDM by the various concentration of BSA, the binding constant was calculated according to the following equation [19]:

$$
\log (1 /[\mathrm{BSA}])=\log K+\log \left(I / I_{0}-I\right)
$$

where, $K$ is the binding constant, $I_{0}$ and $I$ are the peak currents of the free PDM and PDM-BSA complex, respectively. The plot of $\log (1 /[\mathrm{BSA}])$ vs. $\log \left(I /\left(I_{0}-I\right)\right)$ constructed (inset of Fig. 2$)$. From the linear fitting, the binding constant $(K)$ can be estimated from the intercept. With this procedure, we obtained $K=4.591 \times 10^{4} \mathrm{~L} \mathrm{M}^{-1}$ and the correlation coefficient was found as 0.99649 .

\section{Influence of scan rate}

The voltammetric behavior of $1.5 \times 10^{-4} \mathrm{M}$ PDM in the absence and presence of BSA at bare GCE is shown in Fig. 3. The CVs of the $1.5 \times 10^{-4} \mathrm{M}$ PDM on GCE in $0.1 \mathrm{M}$ acetate buffer solution at $\mathrm{pH} 7.2$ shows pair of well defined and stable redox peaks in the potential range of $0.0-1.0 \mathrm{~V}$. An increase in the scan rate, peak potential does not shift but peak currents of both anodic as well as cathodic increased linearly. The scan rate varies from 10 to $400 \mathrm{mV} \mathrm{s}^{-1}$. At the same time, the cathodic and anodic peak currents increased linearly with the scan rate (Fig. 3) and the redox peak currents increased linearly with the scan rate, the correlation coefficient was 0.9919 for cathodic peak and 0.9944 for anodic peak respectively. The redox process is diffusion-controlled as shown by $I_{\text {peak }}$ versus $u^{1 / 2}$ plot inset of Fig. 3 . This was further confirmed by the plot of log $I_{\text {peak }} v s$. log $U$, which was linear within the same scan rate range and gave a slope of 0.5606 [Figure as not shown]. This value is very close to the theoretical value of 0.5 reported by Laviron for a diffusion-controlled 
electrode process [20]. This phenomenon suggested that the redox process was a diffusion controlled and the PDM was stable.

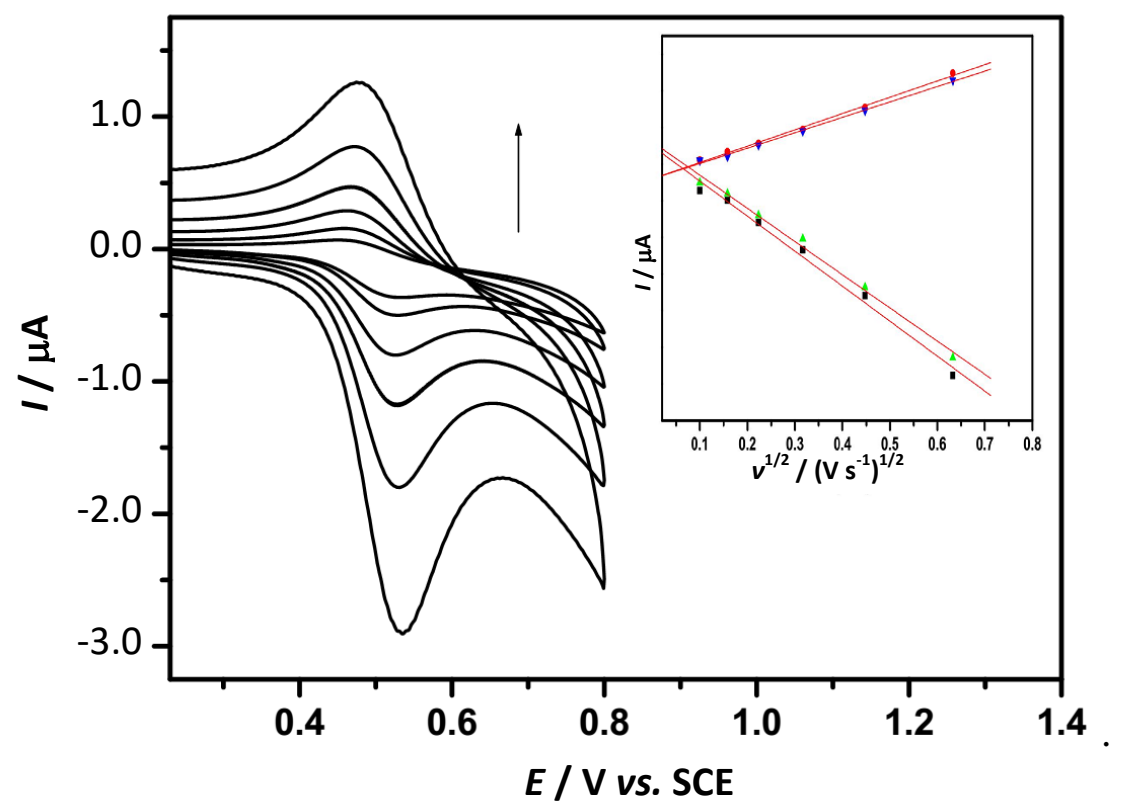

Fig. 3 Cyclic voltammograms of $1.5 \times 10^{-4} \mathrm{M}$ PDM at various scan rates: 10, 25, 50, 100, 200, $400 \mathrm{mV} \mathrm{s}^{-1}$ respectively. Inset, plot of $\mathrm{I}_{\text {peak }}$ versus $\mathrm{U}^{1 / 2}$ (Red and black points are before addition of BSA and blue and green points are after addition of BSA)

The redox peak of PDM is attributed to the one electron which involved reduction and oxidation of PDM to PDM free radical [21].

$$
\text { PDM } \underset{\mathrm{e}^{-}}{\stackrel{-\mathrm{e}^{-}}{\rightleftharpoons}}[\mathrm{PDM}]^{\bullet+}
$$

The electron transfer rate constant $\left(k_{\mathrm{s}}\right)$ is calculated using Laviron's equations [21-23] as follows:

$$
\begin{gathered}
E=E^{0}-2.3 R T \log (v / \alpha \mathrm{n} F) \\
E=E^{O}-2.3 R T \log (v /(1-\alpha) \mathrm{n} F) \\
\log k_{\mathrm{s}}=\log (1-\alpha)+(1-\alpha) \log \alpha-\log (R T / \mathrm{n} F v)-\alpha(1-\alpha) \mathrm{n} F \Delta E_{\mathrm{p}} / 2.303 R T
\end{gathered}
$$

Where $\alpha$ is the electron transfer coefficient, $n$ is the number of electrons transferred $(n=1)$. $R=8.314 \mathrm{~J} \mathrm{~mol}^{-1} \mathrm{~K}^{-1}, T=298 \mathrm{~K}$ and $F=96493 \mathrm{C} / \mathrm{mol}$ are gas, temperature and Faraday constant, respectively and $k_{\mathrm{s}}$ is the electron transfer rate constant which can be calculated according to the plot of $\Delta E_{\mathrm{p}}$ versus In $v$. It is found to be $0.9890 \mathrm{~s}^{-1}$ in absence of BSA and $1.101 \mathrm{~s}^{-1}$ in presence of $B S A$. From the results of the above experiments, the formation of an electrochemically non-active complex of PDM with BSA resulted in the decrease of the free concentration of PDM in the reaction solution which caused the decrease of the peak currents. There is no appreciable difference in the value of $k_{0}$ in presence and absence of BSA indicating that BSA did not alter the voltammetric kinetics of PDM.

\section{Absorption spectral behaviour of PDM with BSA}

The UV-visible absorption titration is simple and more effective method for getting further clues about the mode of interaction and binding strength. Figure. 4 show the electronic absorption spectra of $1.5 \times 10^{-4} \mathrm{M}$ PDM in the absence and presence of different concentrations of BSA. The 
absorbance of PDM was located around at $248 \mathrm{~nm}$. The maximum absorption of the drug at this wavelength exhibited slight bathochromic shift and pronounce hyperchromic shifts by the incremental addition of BSA. The bathochromic shift is associated with the decrease in energy gap between the highest (HOMO) and lowest (LUMO) molecular orbitals after interaction of PDM to BSA [24]. The compactness in the structure of either the drug alone and/or BSA after the formation of drug-BSA complex may result in hyperchromism. Meanwhile, PDM molecule is inserted into the cavities of BSA and disrupted the original structures of BSA. This results which confirms the crystal structure of BSA had changed and there was a strong interaction between PDM and BSA.
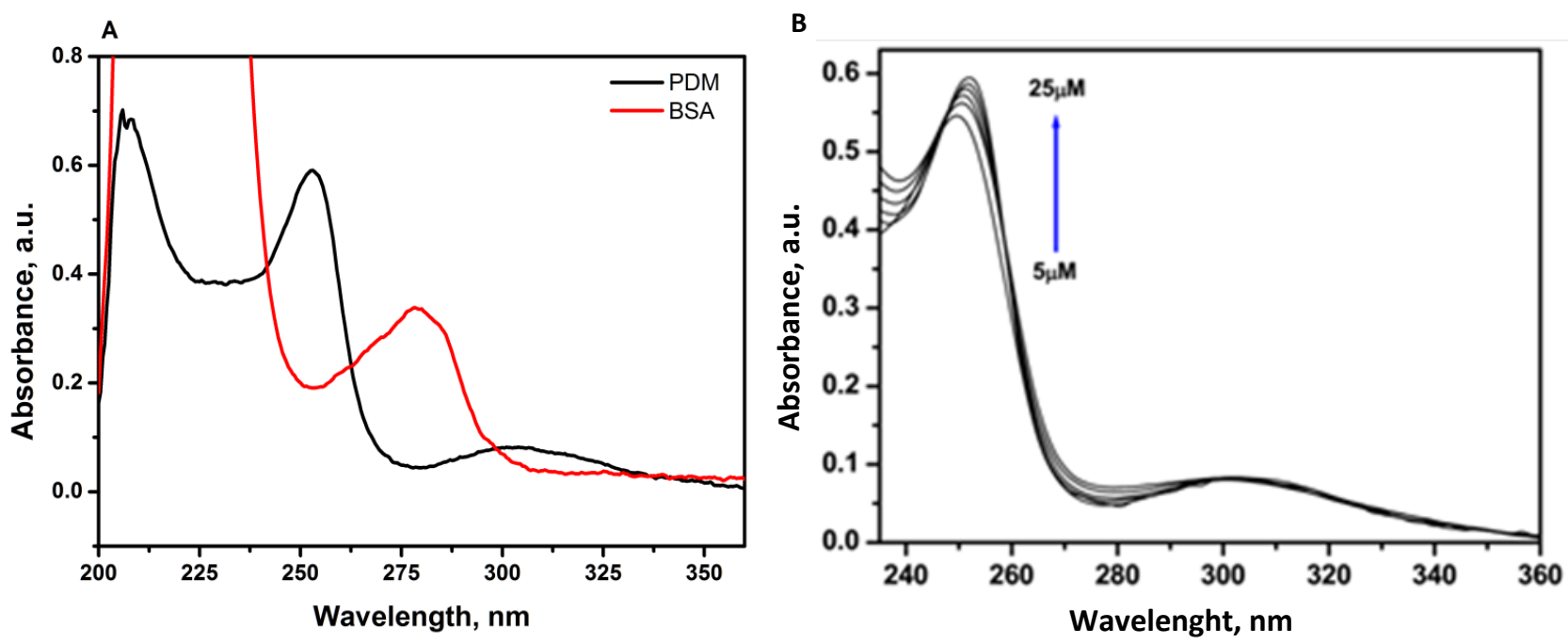

C

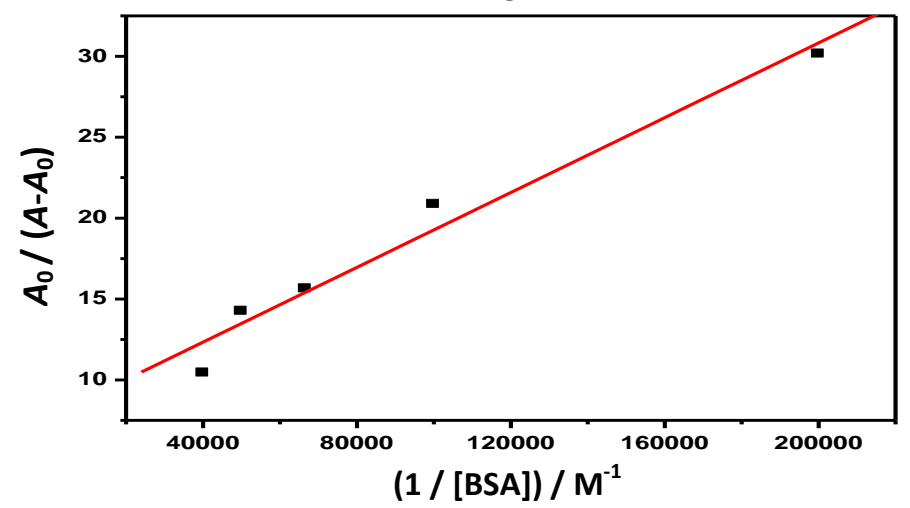

Figure. 4 UV-visible spectra of (A) PDM and BSA, (B) $1.5 \times 10^{-4} \mathrm{M} P D M$ in the absence of BSA and the presence of $[\mathrm{BSA}]=5.0,10.0,15.0,20.0,25.0$ and $30.0 \mu \mathrm{mol} B S A$ in acetate buffer of $\mathrm{pH}$ 7.2. (C) The plot of $\left(\mathrm{A}_{0} /\left(\mathrm{A}-\mathrm{A}_{0}\right)\right.$ versus $1 /$ [BSA].

Based upon the increase in absorbance, the binding constant $\left(K_{\mathrm{b}}\right)$ was calculated according to the following equation [24].

$$
A_{0} /\left(A-A_{0}\right)=\varepsilon_{\mathrm{G}} /\left(\varepsilon_{\mathrm{H}-\mathrm{G}}-\varepsilon_{\mathrm{G}}\right)+\varepsilon_{\mathrm{G}} /\left(\varepsilon_{\mathrm{H}-\mathrm{G}}-\varepsilon_{\mathrm{G}}\right) \times 1 / K[\mathrm{BSA}]
$$

Where, $A_{0}$ and $A$ are the absorbance of PDM in the absence and presence of BSA, $\varepsilon_{\mathrm{G}}$ and $\varepsilon_{\mathrm{H}-\mathrm{G}}$ are the absorption coefficients of PDM and its complex with BSA, respectively.

The slope to intercept ratio of the plot between $A_{0} /\left(A-A_{0}\right)$ vs. $1 /$ [BSA] yielded the binding constant, $K=6.6671 \times 10^{4} \mathrm{~L} \mathrm{M}^{-1}$, which is close to the value of $K=1.9824 \times 10^{4} \mathrm{~L} \mathrm{M}^{-1}$ obtained from $\mathrm{CV}$. The moderate binding constant indicative of electrostatic interaction and the Gibbs energy 
change $(\Delta G=-R T \ln K)$ of approximately $24.039 \mathrm{~kJ} \mathrm{~mol}^{-1}$ at $25^{\circ} \mathrm{C}$ signifies the spontaneity of interaction of PDM with BSA.

The type of quenching mechanism was determined using UV-visible absorption spectroscopy. Quenching usually occurs either by static or dynamic mode. Static quenching refers to fluorophore-quencher complex formation and the dynamic quenching refers to a process that the fluorophore and the quencher come into contact during the transient existence of the excited state. However, the formation of non-fluorescence ground-state complex induced the change in absorption spectrum of fluorophore. Addition of the BSA to PDM leads to an increase in PDM absorption intensity without affecting the position of absorption band (Fig. 4A). It showed the existence of static interaction between BSA and the complexes.

\section{Spectrofluorimetric spectral behaviour of PDM-BSA complex}

The interaction of PDM with BSA was also examined by fluorescence titration. The fluorescence emission spectra of BSA in the presence and absence of various concentration of PDM is shown in Fig. 5. The maximum emission of BSA is located at $342 \mathrm{~nm}\left(\lambda_{\mathrm{ex}}=263 \mathrm{~nm}\right)$. The fluorescence emission maxima were gradually decreased with increase in concentration of PDM, indicating the quenching of fluorescence intensity of BSA upon binding to PDM. This can be attributed to the formation of PDM-BSA complex. The results suggest that microenvironment around the chromospheres of BSA is changed and it shows the strong interaction between PDM and BSA [25].

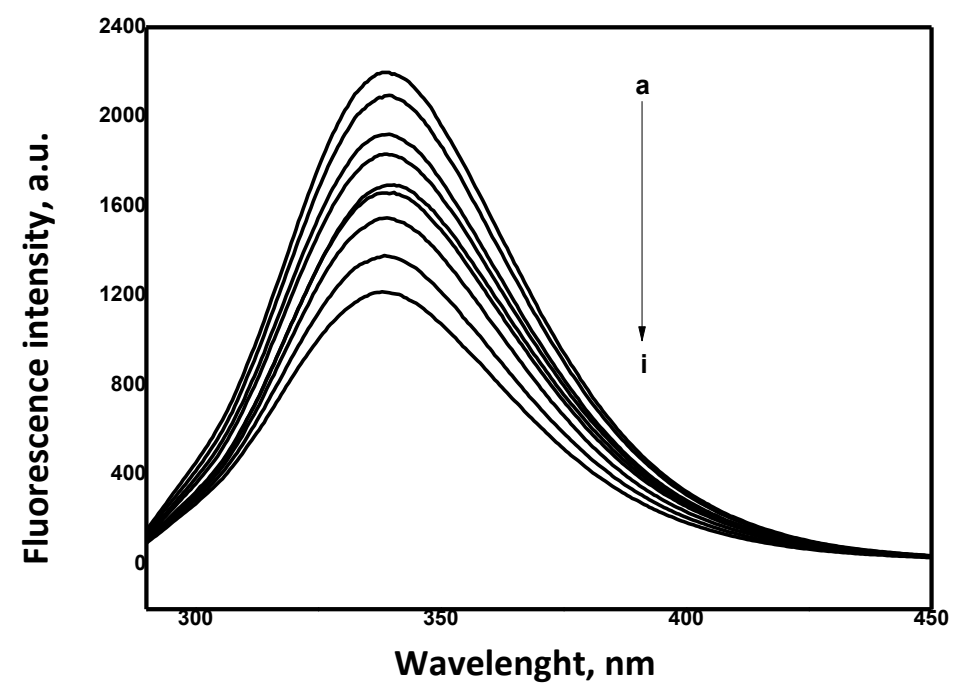

Fig. 5. Fluorescence spectra of $1.5 \times 10^{-4} \mathrm{M} B S A$ in presence of $C_{P D M}=0,5,10,15,20,25,30,35 \mu \mathrm{mol} L^{-1}$.

\section{Stern-Volmer binding and quenching constant}

To determine the quenching mechanism between BSA and PDM (i.e. dynamic or static quenching), the fluorescence quenching data were analyzed using Stern-Volmer equation.

$$
F_{0} / F=1+k_{\mathrm{q}} \tau_{\mathrm{o}}[\mathrm{Q}]=1+K_{\mathrm{sv}}[\mathrm{Q}]
$$

where, $F_{0}$ and $F$ are the fluorescence intensities of BSA in absence and presence of PDM respectively, [Q] is the Concentration of $\mathrm{BSA}$ and $k_{\mathrm{q}}$ is the quenching rate constant of the biomolecule, $\tau_{\mathrm{o}}$ is the average excited-state life time and $K_{\mathrm{sv}}$ is the Stern-Volmer quenching constant. 

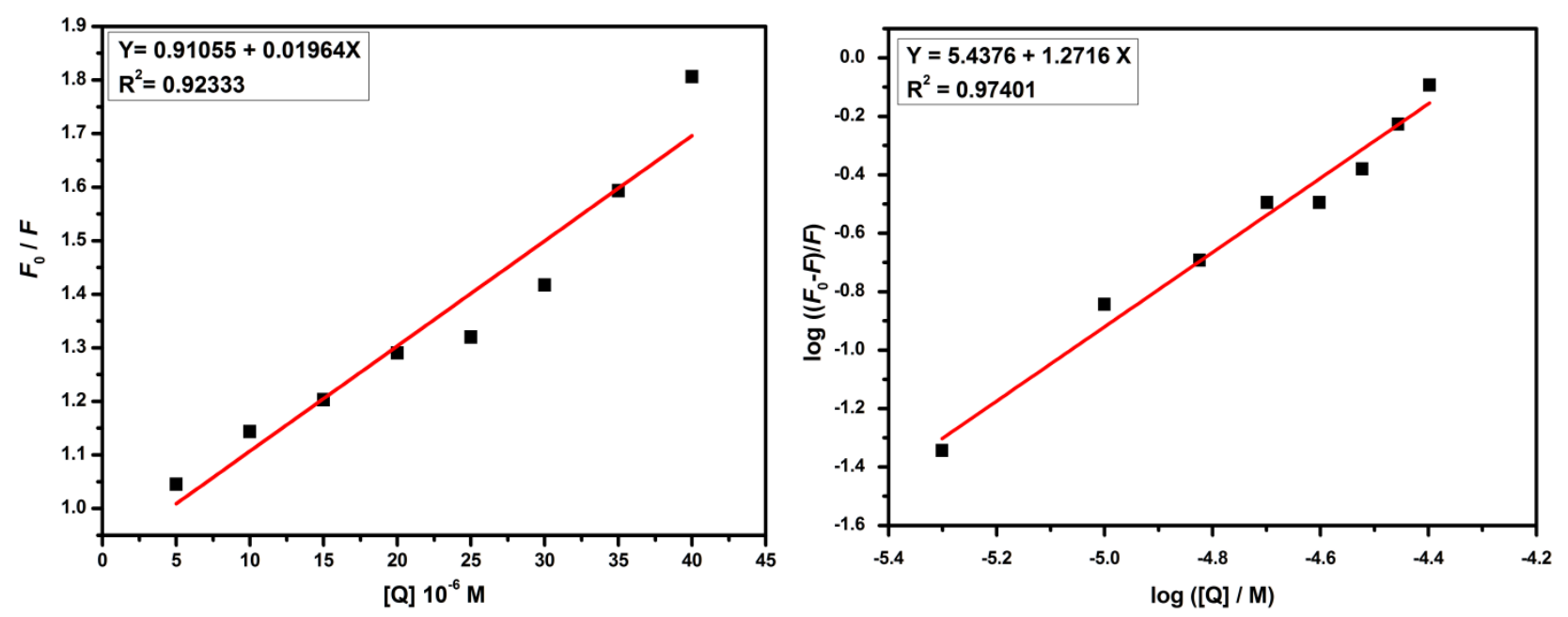

Fig. 6 (a) Plot of $\mathrm{F}_{0} / \mathrm{F}$ versus $[\mathrm{Q}]$ and (b) Plot of $\log \left[\left(\mathrm{F}_{0}-\mathrm{F}\right) / \mathrm{F}\right]$ versus $\log [\mathrm{Q}]$

The $K_{\mathrm{sv}}$ is stern-volmer quenching constant, which was determined by linear regression of a plot of $F_{0} / F$ vs. [Q] at $25{ }^{\circ} \mathrm{C}$ was constructed (Fig. 6a) using the data from fluorescence titration and a linear fitting of the data yielded the binding constant $2.4158 \times 10^{4} \mathrm{~L} \mathrm{M}^{-1}$ which is good agreement with result obtained from CV and UV/vis spectroscopy. The maximum rate constant of collisional quenching of various quenchers with biopolymers is about $2.0 \times 10^{10} \mathrm{~L} \mathrm{~s}^{-1} \mathrm{~mol}^{-1}$ [25]. The greater value for $K_{\mathrm{q}}$ is obtained ( $K_{\mathrm{sv}}$ and $k_{\mathrm{q}}$ values calculated were $1.964 \times 10^{4} \mathrm{~L} \mathrm{M}^{-1}$ and $1.964 \times 10^{12} \mathrm{~L} \mathrm{M}^{-1} \mathrm{~s}^{-1}$ ) $\left(R^{2}=0.99523\right)$ respectively. As temperature increased, the decreasing trend of $K_{q}$ was in accordance with $K_{s v}$ 's dependency on temperature, which coincided with a static quenching mechanism. The binding of PDM to BSA was reduced as temperature increased, which suggests that the fluorescence quenching process may be mainly controlled by static quenching mechanism rather than dynamic.

\section{The binding constant and number of binding sites}

The binding constant and number of binding sites for PDM-BSA were determined by the following equation [26]:

$$
\log \left[\left(F_{0}-F\right) / F\right]=\log K_{\mathrm{b}}+\mathrm{n} \log [\mathrm{Q}]
$$

where, $K_{\mathrm{b}}$ is binding constant and $\mathrm{n}$ is number of binding sites in base pair units respectively. The values of $\mathrm{n}$ and $K_{\mathrm{b}}$ can be determined from the slope and intercept of the double logarithm regression curve $\log \left[\left(\log \left[\left(F_{0}-F\right) / F\right]\right.\right.$ vs. $\left.\log [Q]\right)$ (Fig. $\left.6 \mathrm{~b}\right)$. The binding constant value $5.43 \times 104 \mathrm{~L} \mathrm{M}^{-1}$ $\left(R^{2}=0.99879\right)$ and values of $\mathrm{n}$ is found to be $1.2 \approx 1.0$, indicating that there is one independent class of binding sites in BSA for PDM. The free energy change, $\Delta G^{0}$ (at $27^{\circ} \mathrm{C}$ ) was evaluated from $K_{\mathrm{b}}$ using the relationship $\Delta G^{0}=-2.303 R T \log K_{\mathrm{b}}$ and its value was found to be $-27.016 \mathrm{~kJ} \mathrm{M}^{-1}$ indicating the spontaneity of the reaction.

\section{Molecular modeling studies}

Molecular docking was performed to study the interaction between PDM and BSA to determine the preferred binding site and binding mode. The best confirmation was determined based on binding affinity and RMSD. The binding energy and RMSD was performed by Auto Dock Vina. A three dimensional docked structure of PDM on BSA was shown in Fig. 7. These structures were to study various inter molecular interaction and to determine the binding energy of the docked 
complexes. The PDM were docked with receptor BSA using the parameters mentioned above. The energy of interaction for PDM-BSA complex was found to be $-10.0 \mathrm{kcal} \mathrm{mol}^{-1}$.
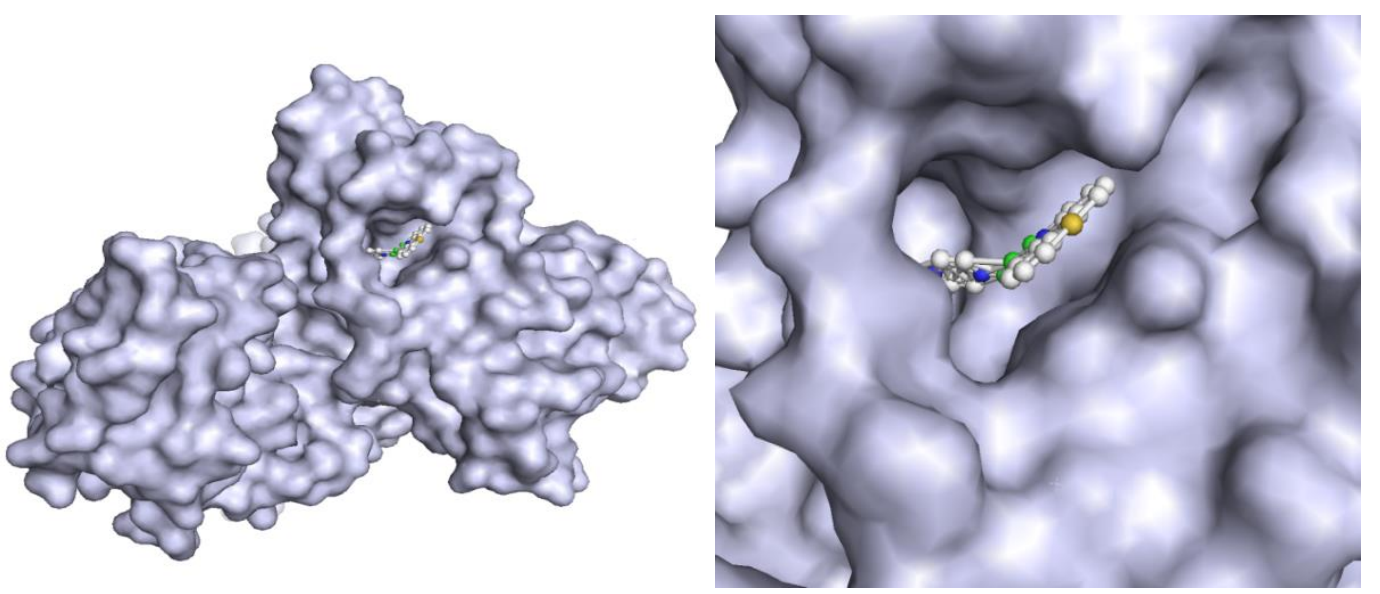

Fig. 7. Molecular model of the surface and the active site of the complex of PDM (stick) and BSA (white surface)

\section{Viscometer measurement}

Viscometric technique is an effective tool in clarifying the mode of interaction of small molecules with BSA under the physiological condition. In general, intercalation (in-binding mode) causes an increase viscosity of BSA solution due to lengthening the BSA widened to accommodate the bonded molecule [27]. The reverse can be taken for the electrostatic interaction. The value of relative specific viscosity $\left(\eta / \eta_{0}\right)^{1 / 3}$ vs. [BSA]/[PDM] were plotted (Fig. 8). However, the relative viscosity of BSA dependence upon the concentration of PDM, the value of [BSA]/[PDM] is decreased. This behaviour indicates that the binding mode between BSA and PDM should be nonclassical intercalation or groove mode via hydrophobic interaction.

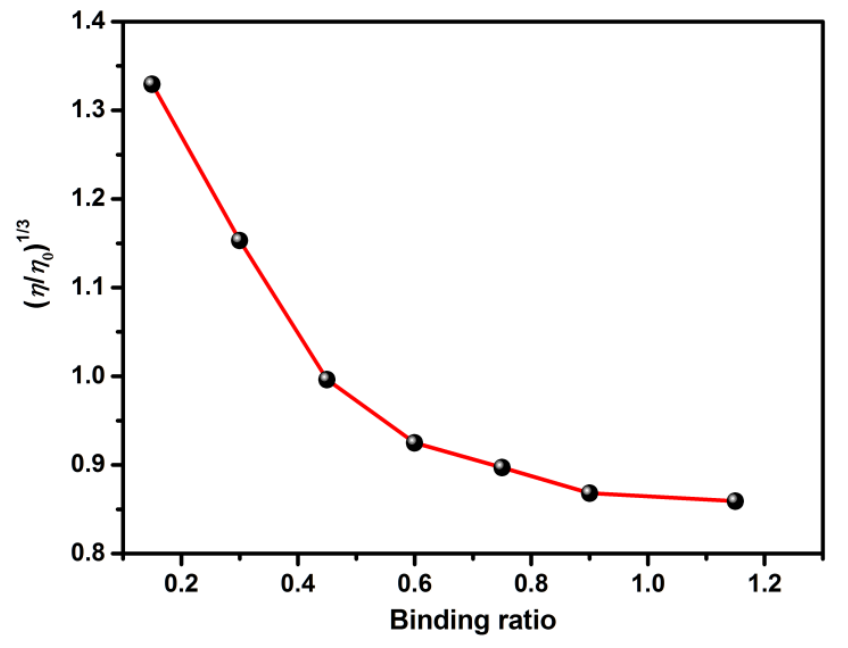

Fig. 8. Plot of $[B S A / P D M]$ vs. $\left(\mathrm{\eta} / \mathrm{n}_{0}\right)^{1 / 3}$

\section{Conclusions}

In this work, the interaction of PDM with BSA was studied by voltammetric, spectroscopic and viscometric methods. In voltammetric studies, it was observed that the presence of BSA in a solution of perazine dimalaete reduces the equilibrium concentration of free perazine dimalaete and produces a voltammetric inactive complex. Both electrostatic interactions and minor groove binding modes were deduced from the results of different methods applied to the system, 
although groove binding seemed to be predominant. Meanwhile, the experimental results indicate that the quenching mechanism of fluorescence of BSA by PDM is a static quenching procedure and the binding reaction is spontaneous. This work provides some significant information to clinical research about PDM and the theoretical basis for new drug designing.

Acknowledgement: The authors are grateful for the financial support provided by the University Grants Commission, New Delhi, India (F. No. 42-308/2013 (SR) Dated 28/03/2013). Thanks are also due to Global Calcium Ltd., Hosur for supplying gift samples of PDM. Thanks are also due to Dr. Siddalingeshwar, Department of Physics, MSRIT, Bangalore for providing spectrofluorimetric instrumental facility

\section{References}

[1] E. La Zaro, P. J. Lowe, X. Briand, B. Faller, J. Med. Chem. 51 (2008) 2009-2017.

[2] B. Ahmad, S. Parveen, R.H. Khan, Bio-macromolecules 7 (2006) 1350-1356.

[3] F. Bosca, J. Phys. Chem. B 116 (2012) 3504-3511.

[4] R. E. Olson, D.D. Christ, Ann. Rep. Med. Chem. 31 (1996) 327-336.

[5] E. Ahmad, G. Rabbani, N. Zaidi, S. Singh, M. Rehan, M. M. Khan, S. K. Rahman, Z. Qadri, M. Shadab, M. T Ashraf, N. Subbarao, R. Bhat, R. H. Khan, PLoS One 6(11) (2011) e26186 1-18.

[6] E. Ahmad, P. Sen, R.H. Khan, Cell. Biochem. Biophys. 61 (2011) 313-325.

[7] G. K Wang, X. Li, X. L. Ding, D. C. Wang, C. L Yan, Y. Lu, J. Pharm. Biomed. Anal. 55 (2011) 1223-1226.

[8] F. P Nicoletti, B. D. Howes, M. Fittipaldi, G. Fanali, M. Fasano, P. Ascenzi, G Smulevich, J. Am. Chem. Soc. 130 (2008) 11677-11688.

[9] F. Thomas, P. Rochaix, M. White-Koning, I. Hennebelle, J. Sarini, A. Benlyazid, L. Malard, J. Lefebvre, E. Chatelut, J. P. Delord, Eur. J. Cancer. 45 (2009) 2316- 2323.

[10] B. M. Zeglis, V. C. Pierre, J. K. Barton, Chem. Commun. 44 (2007) 4565-4579.

[11] Q. Lu, D.Y. Qing, Sci. China. Life Sci. 56 (2013) 1020-1027.

[12] M. E. Reichmann, S. A. Rice, C. A. Thomas, P. Doty, J. Am. Chem. Soc. 76 (1954) 3047.

[13] G. M. Morris, D. S. Goodsell, R. S. Halliday, J. Comput. Chem. 19 (14) (1998) 1639-1662.

[14] A. T. Laurie, R. M. Jackson, Bioinformatics 21(9) (2005) 1908-1916.

[15] N. M. O'Boyle, M. Banck, J. Chem. Inform. 3 (2011) 33.

[16] O. Trott, A. J. Olson, J. Comput. Chem. 31 (2010) 455-461.

[17] M. Aslanoglu, Anal. Sci 22 (2006) 439.

[18] N. Li, Y. Ma, C. Yang, L. Guo, X. Yan, Biophys. Chem. 116 (2005) 199.

[19] F. Jalali, P. S. Dorraji, J. Pharma. Biomed. Anal. 70 (2012) 598-601.

[20] E. Laviron, J. Electroanal. Chem. 101 (1979) 19-28.

[21] G. C. Zhao, J. J. Zhu, J. J. Zhang, H. Y. Chen, Anal. Chim. Acta 394 (1999) 337.

[22] N. Alexei, M. Pankratov, I. Uchaeva, N. A. Stepanov, Can. J. Chem. 71 (1993) 674-677.

[23] B. Xu, K. Jiao, Y. Ren, W. Sun, J. Chin. Chem. Soc. 54 (2007) 917-924.

[24] A. Shah, M. Zaheer, R. Qureshi, Z. Akhter, M. F. Nazar, Spectrochimica Acta Part A 75 (2010) 1082-1087.

[25] J. Jayabharathi, V. Thanikachalam, M. Venkatesh Perumal, N. Srinivasan, Spectrochim. Acta Part A: Mol. Biomol. Spectr. 79 (2011) 236

[26] C. Wang, Q. Chu, C. Chen, Z. Bo, Spectroscopy 25 (2011) 113-122.

[27] Q. Wang, X. Wang, Z. Yu, X. Yuan, K. Jiao, Int. J. Electrochem. Sci. 6 (2011) $5470-5481$.

(c) 2016 by the authors; licensee IAPC, Zagreb, Croatia. This article is an open-access article distributed under the terms and conditions of the Creative Commons Attribution license

(http://creativecommons.org/licenses/by/4.0/) 